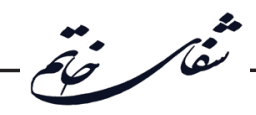

\title{
The Role of Religious Coping, Spiritual Intelligence, and Spiritual Well-being in Predicting the Perceived Stress of Patients with Cancer
}

\author{
Sanaz Einy ${ }^{1^{*}}$, Zohre Hashemi ${ }^{2}$ \\ ${ }^{1}$ Department of Psychology, Faculty of Educational Sciences and Psychology, University of Mohaghegh Ardabili, Ardebil, Iran \\ ${ }^{2}$ Department of Psychology, University of Maragheh, East Azarbaijan, Iran
}

\section{ABSTRACT}

Introduction: One of the main issues facing the patients with cancer is stress and anxiety. On the other hand, religion and spiritual resources are very effective factors in mental health. The purpose of this study was to investigate the role of religious coping, spiritual intelligence, and spiritual well-being in predicting perceived stress in patients with cancer. Materials and Methods: In this descriptive cross-sectional study, 120 patients, with cancer who were admitted and treated in blood and oncology department of Imam Khomeini hospital of Ardabil in 2019, were selected as target sample. Data collection tools consisted of perceived stress scale, religious coping scale, spiritual intelligence scale, and spiritual well-being questionnaire. Results: There was a negative and significant relationship between the perceived stress with the spiritual well-being $(\beta=-0 / 359$ : $p<0 / 001)$, spiritual intelligence $(\beta=-0 / 170$ : $p<0 / 007)$, positive religious coping $(\beta=-0 / 172: p<0 / 011)$ and positive and significant relationship with negative religious coping $(\beta=0 / 328$ : $p<0 / 001)$ in subjects with cancer. The spiritual well-being, religious coping and spiritual intelligence predicted $70 \%$ of variance of perceived stress scores of patients with cancer $(p<0 / 01)$. Conclusion: Our findings indicate that positive and negative religious coping, spiritual intelligence, and spiritual well-being are associated with perceived stress and can explain one's attitude toward illness. Therefore, training patients to empower them in these areas have beneficial effects on their health-related quality of life.

\section{Key words:}

1. Adaptation, Psychological

2. Intelligence

3. Patients

*Corresponding Author: Sanaz Einy

E-mail: sanaz.einy@yahoo.com 
نقش مقابلهُ مذهبى، هوش معنوى و بهزيستى معنوى در ييشبينى استرس ادراكشدهُ بيماران مبتلا به سرطان

$$
\text { ساناز عينى ":" زهره هاشمى }
$$

'كروه روانشناسى، دانشكده علوم تربيتى و روانشناسى، دانشكاه محقق اردبيلى، اردبيل، ايران

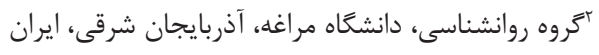

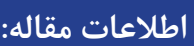

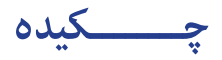

مقدمه: يكى از عمدهترين مسائلى كه بيماران مبتلا به سرطان بان با آن مواجهاند، استرس و اضطرابر إبراب

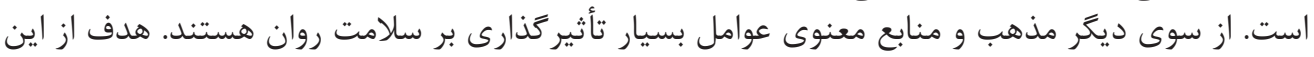

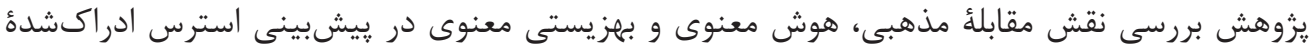

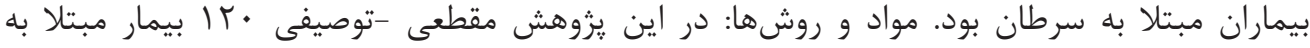

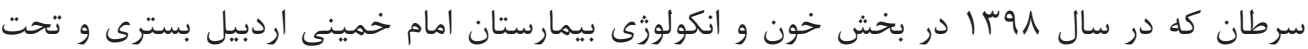

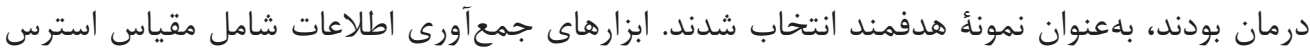

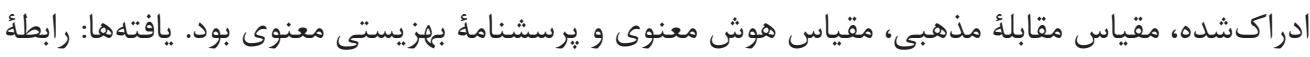

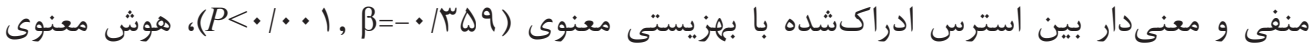

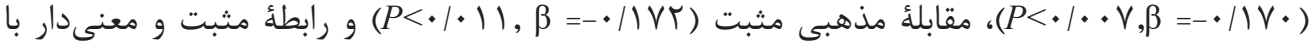

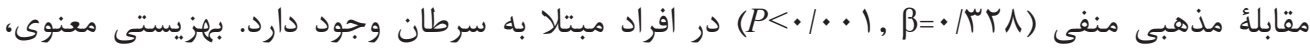

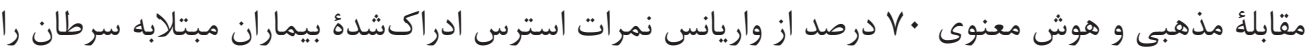

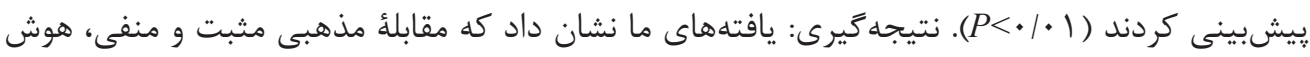

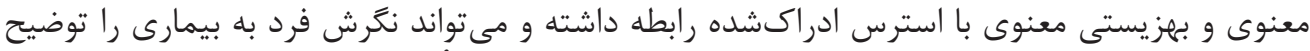

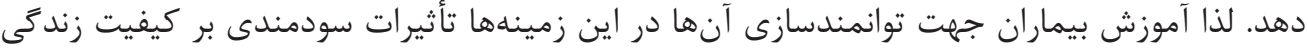

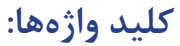
مرتبط با سلامت آنها دارد.

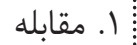

r ب.

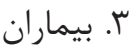

" نويسنده مسئول: ساناز عينى آدرس الكترونيكى: sanaz.einy@yahoo.com 


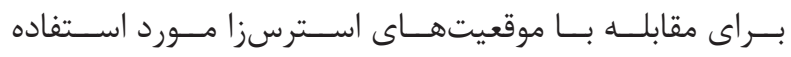

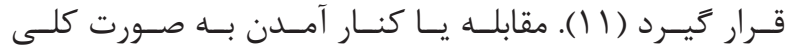

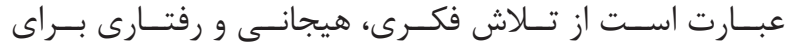

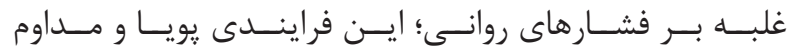

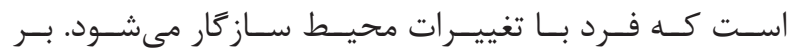

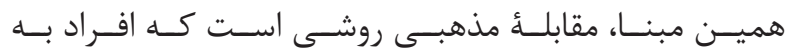

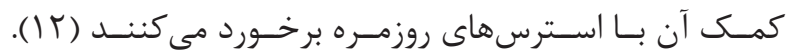

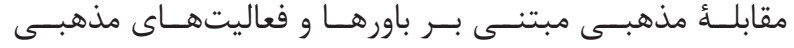

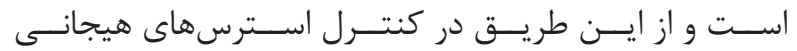

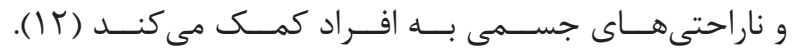

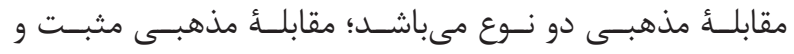

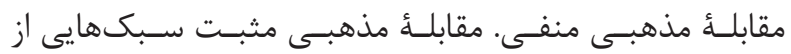

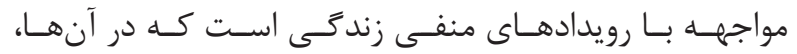

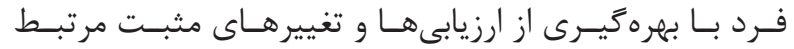

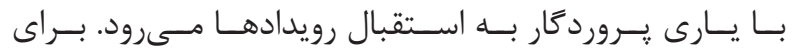

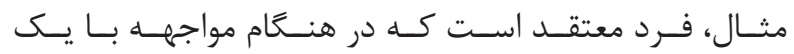

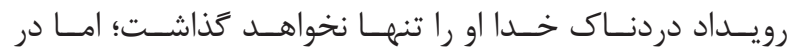

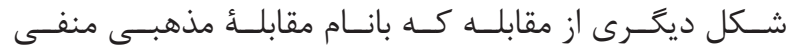

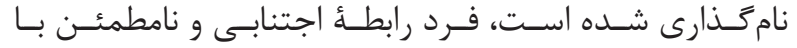

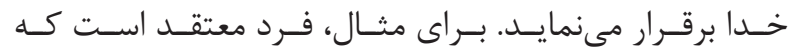

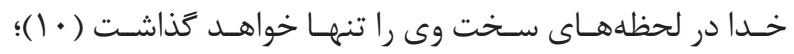

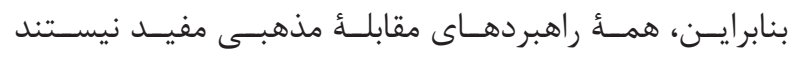

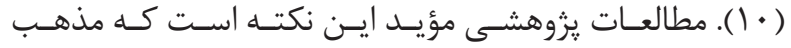

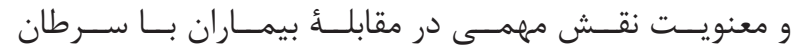

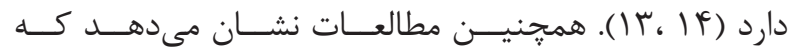

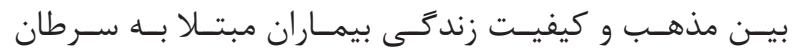

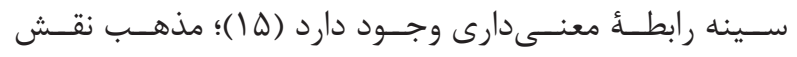

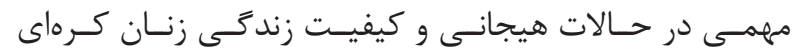

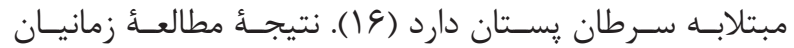

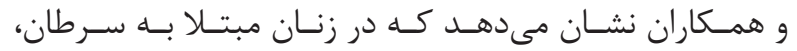

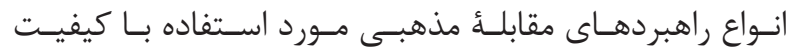

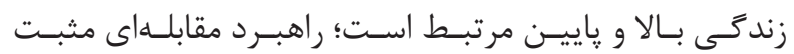

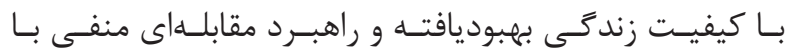

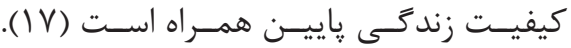

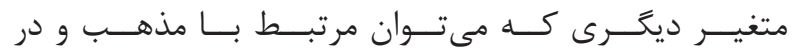

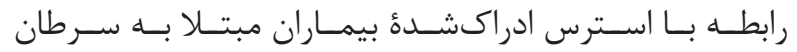

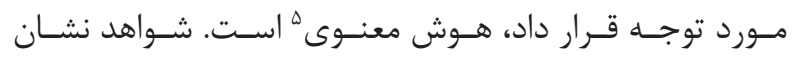

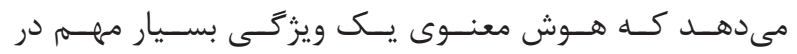

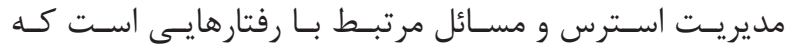

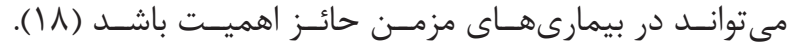

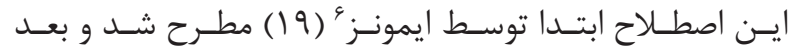

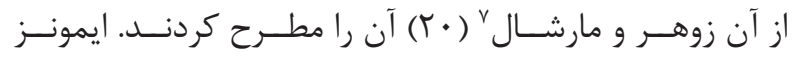

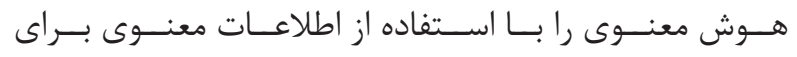

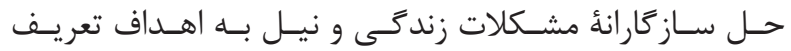

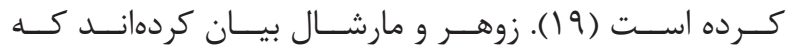

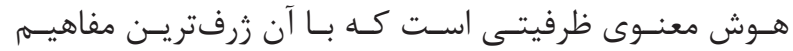

${ }^{1}$ Perceived stress

${ }^{2}$ Cognitive-interactive model of stress

${ }^{3}$ Pargament

${ }^{4}$ Religious coping

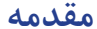

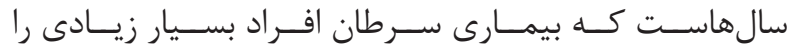

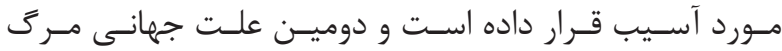

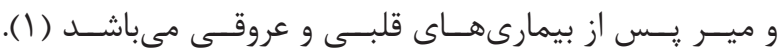

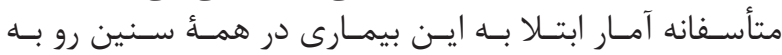

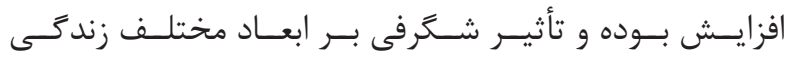

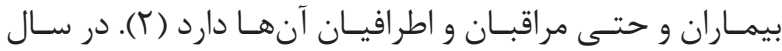

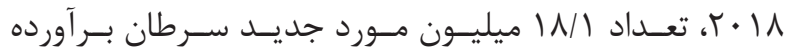

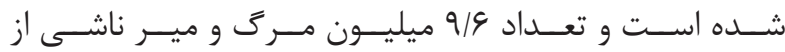

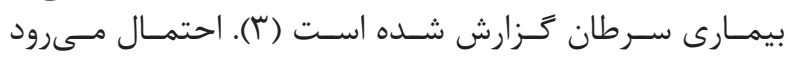

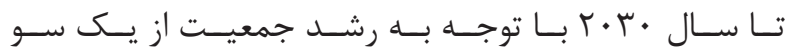

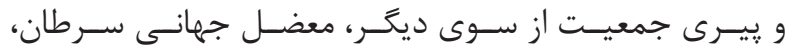

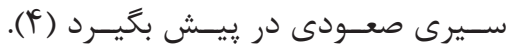

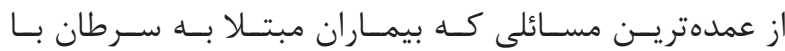

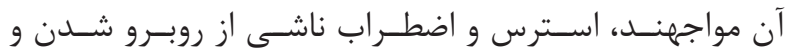

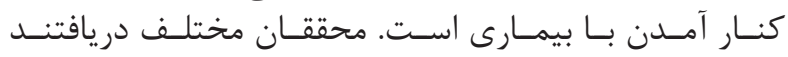

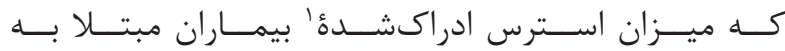

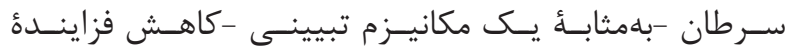

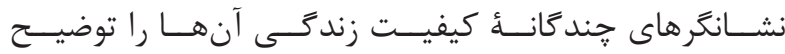

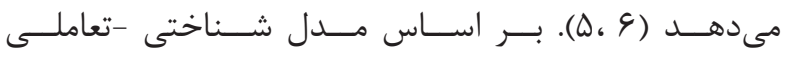

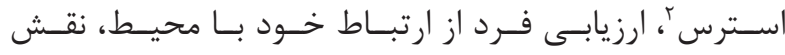

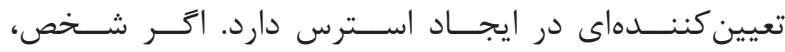

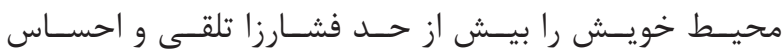

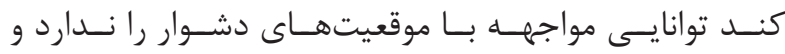

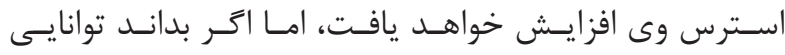

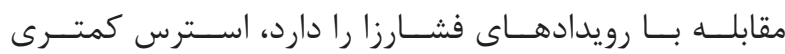

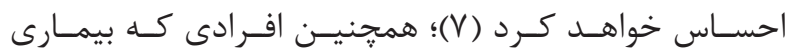

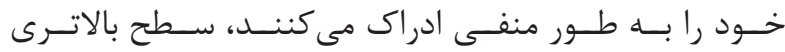

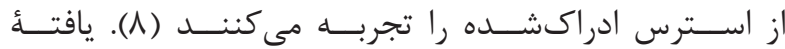

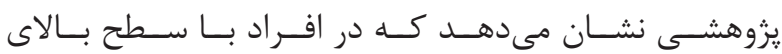

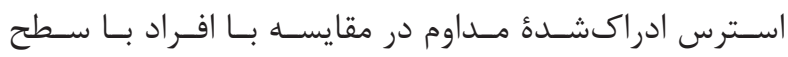

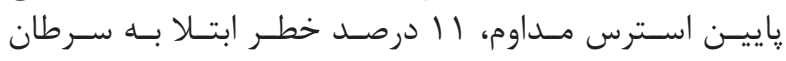

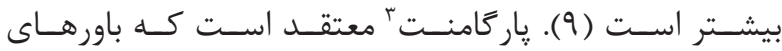

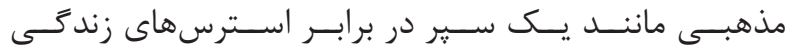

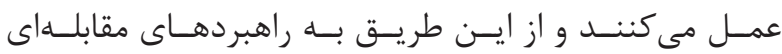

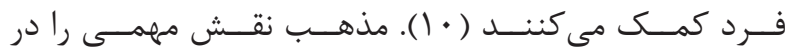

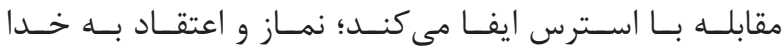

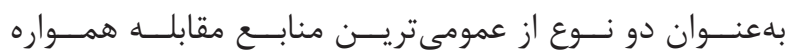

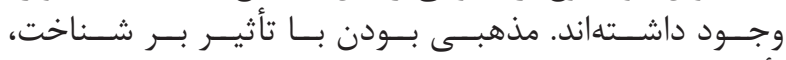

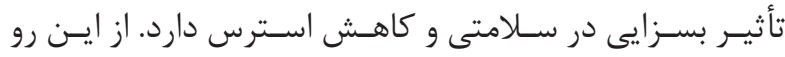

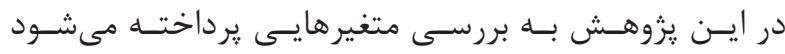

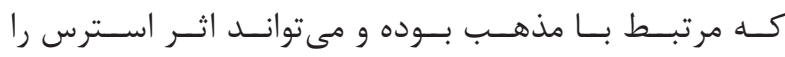

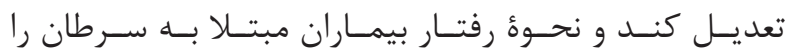

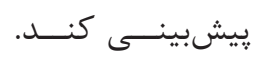

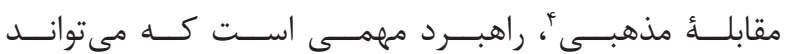

${ }^{5}$ Spiritual intelligence

${ }^{6}$ Emmons

${ }^{7}$ Zohar and Marshall 


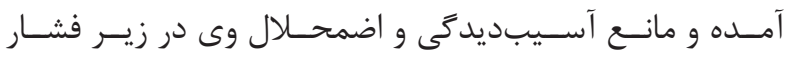

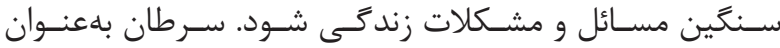

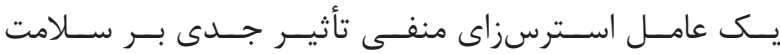

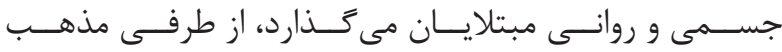

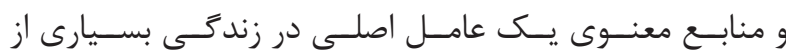

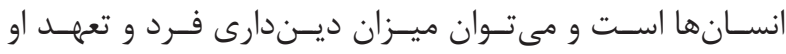

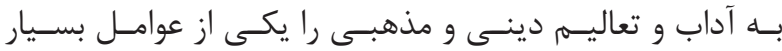

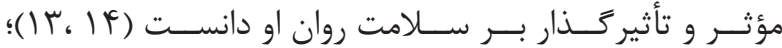

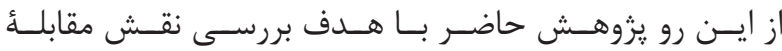

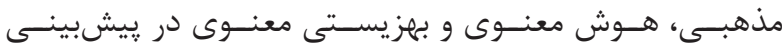

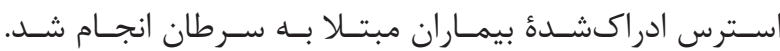

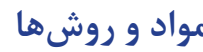

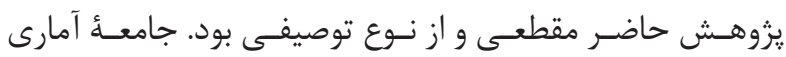

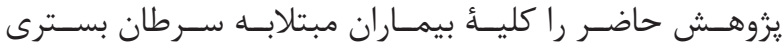

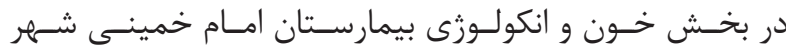

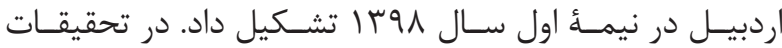

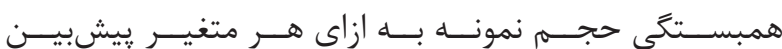

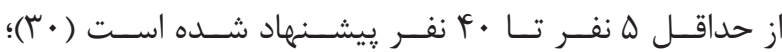

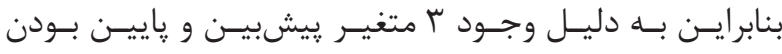

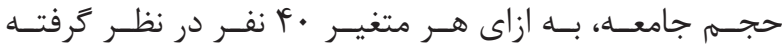

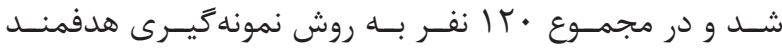

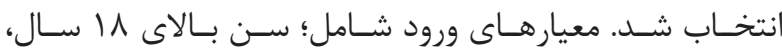

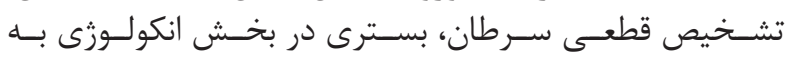

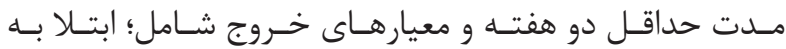

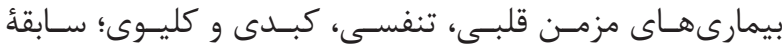

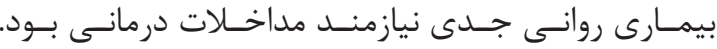

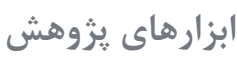

\section{مقياس استرس ادراكشده}

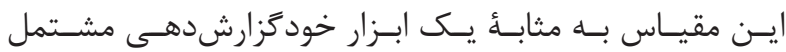

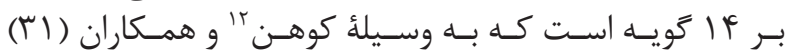

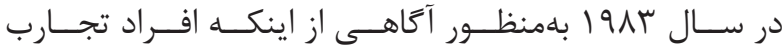

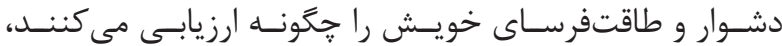

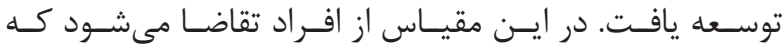

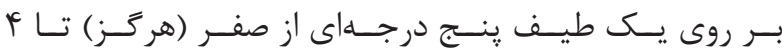

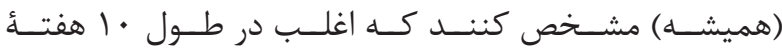

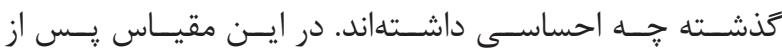

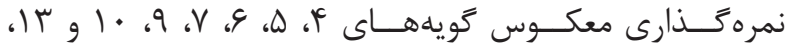

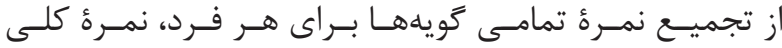

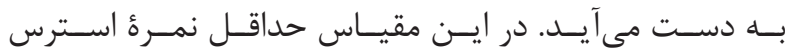

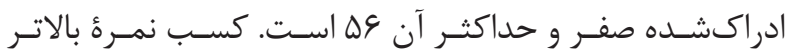

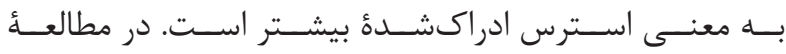

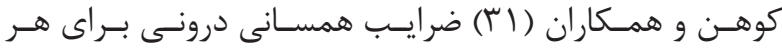

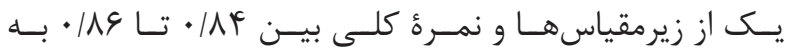

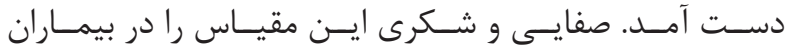

${ }^{8}$ Spiritual well-being

${ }^{9}$ Religious well-being

${ }^{10}$ Existential well-being

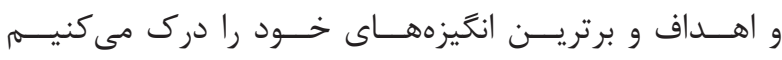

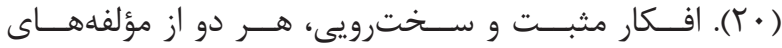

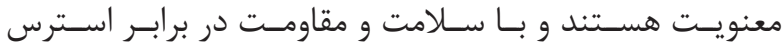

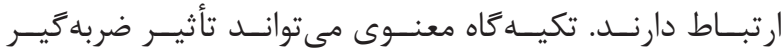

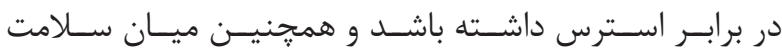

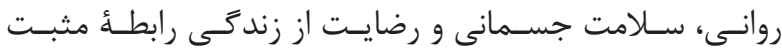

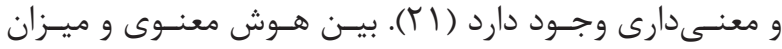

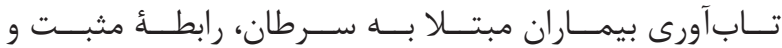

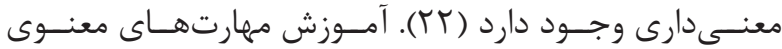

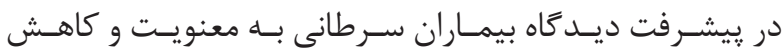

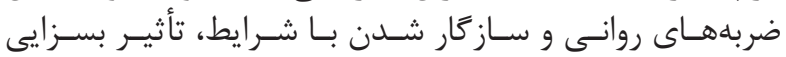

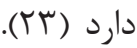

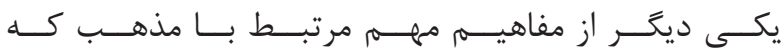

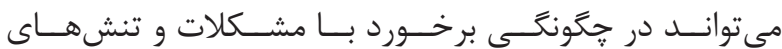

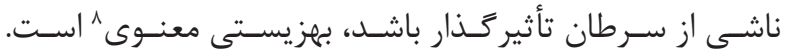

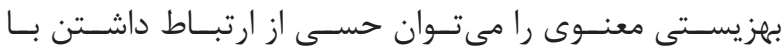

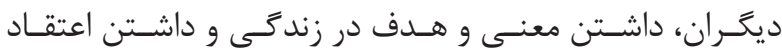

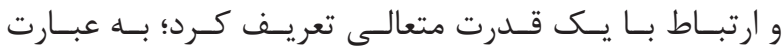

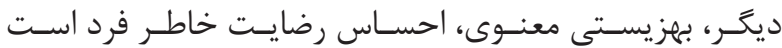

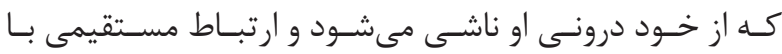

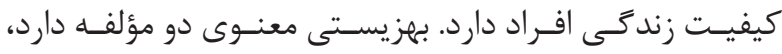

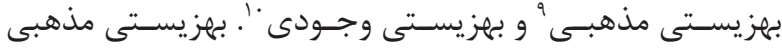

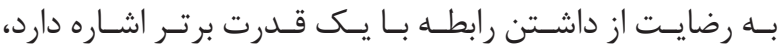

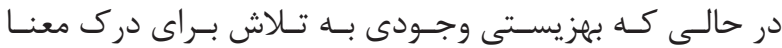

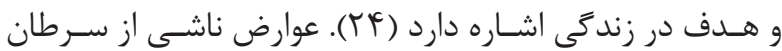

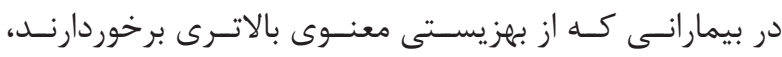

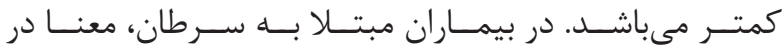

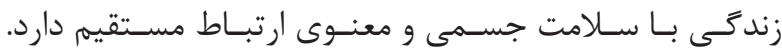

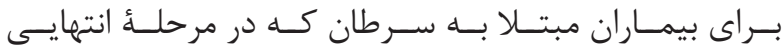

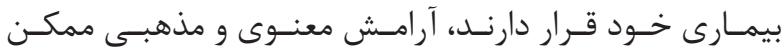

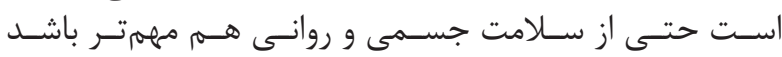

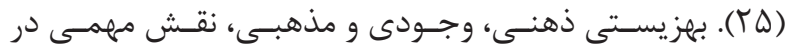

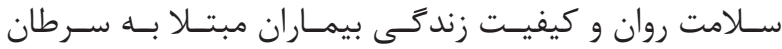

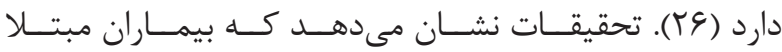

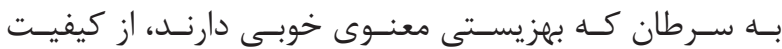

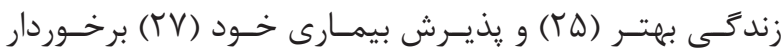

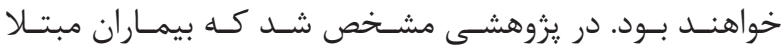

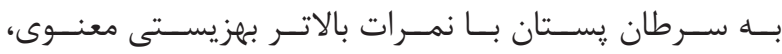

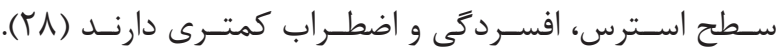

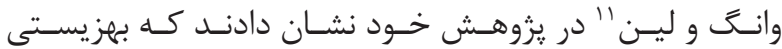

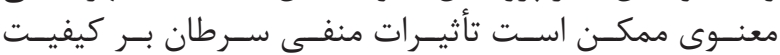

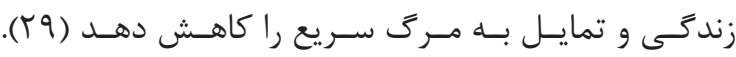

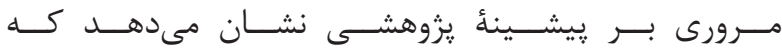

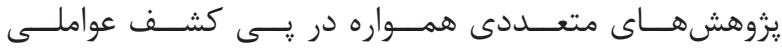

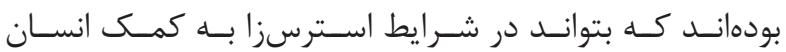

${ }^{11}$ Wang and Lin

${ }^{12}$ Cohen 


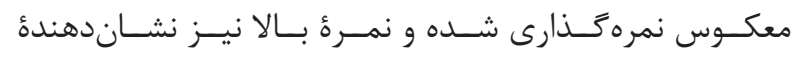

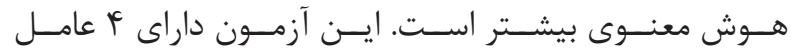

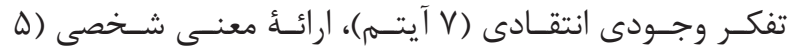

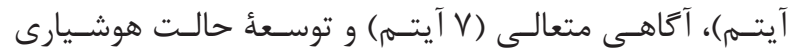

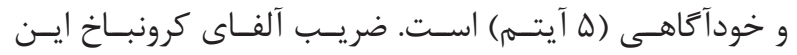

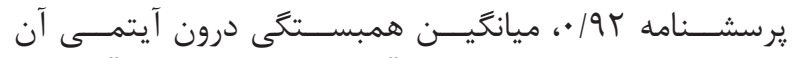

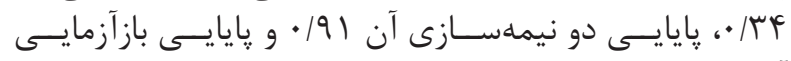

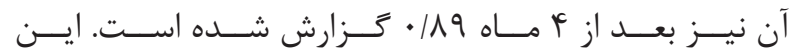

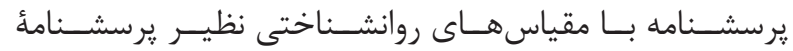

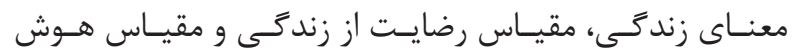

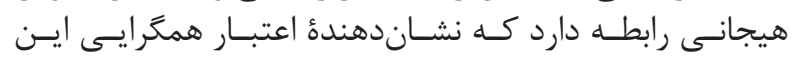

يرسشـنامه اسـت (YT)

\section{آزمون بهزيستى معنوى}

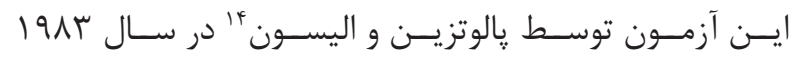

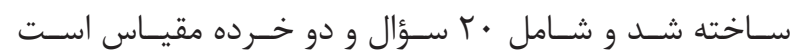

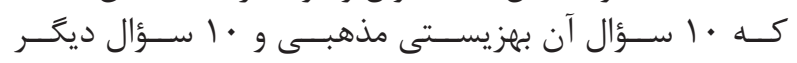

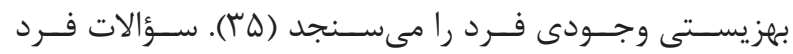

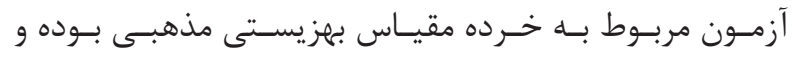

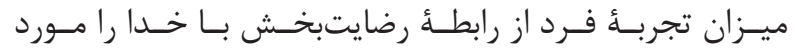

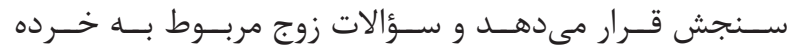

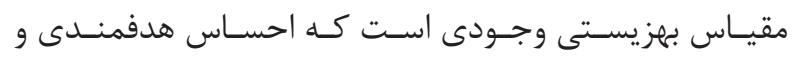

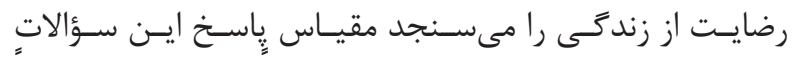

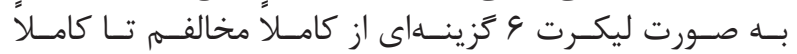

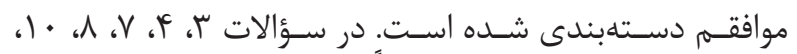

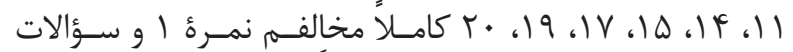

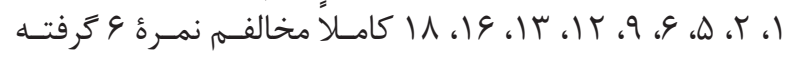

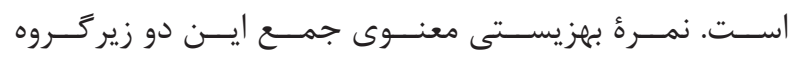

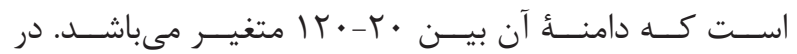

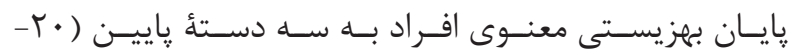

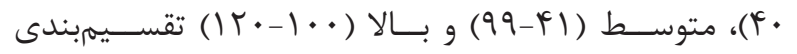

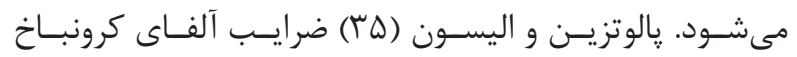

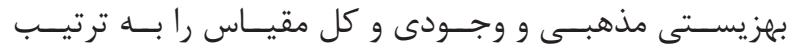

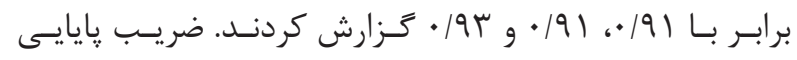

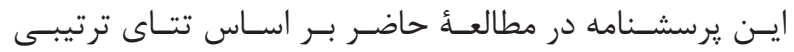

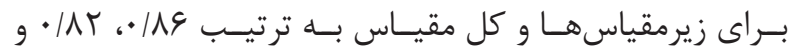

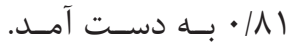

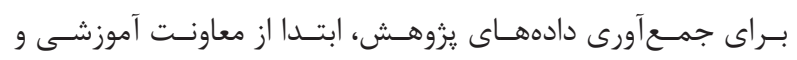

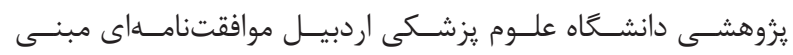

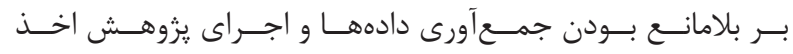

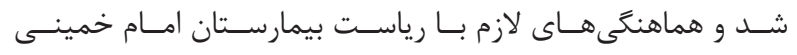

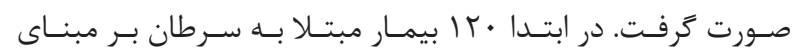

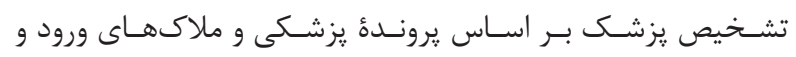

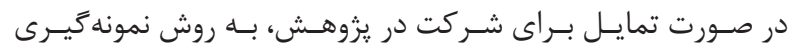

هدفمنــد انتخـاب شـد

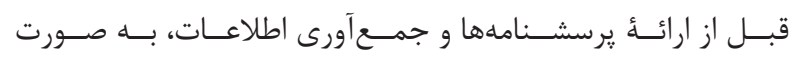

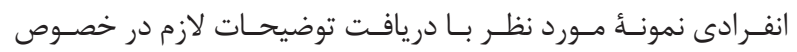

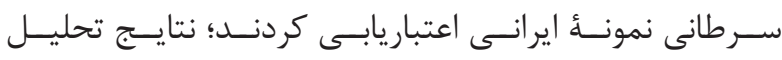

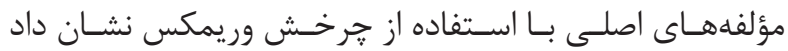

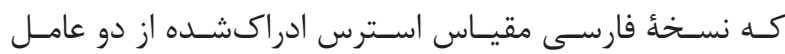

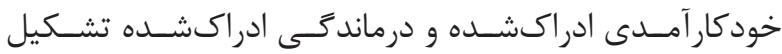

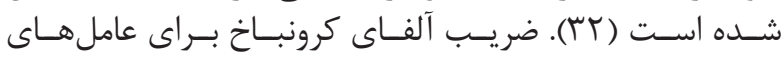

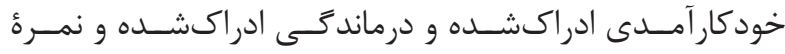

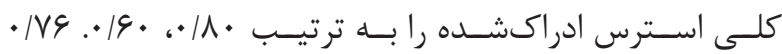
بـه دسـت آوردنـد.

\section{نسخدُ كو تاه مقياس مقابلة مذهبى}

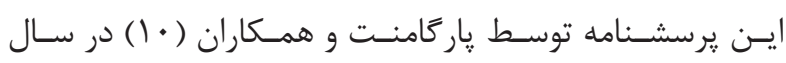

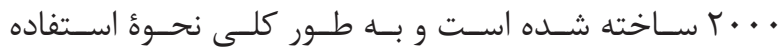

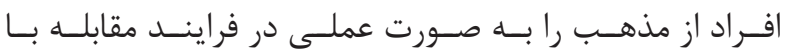

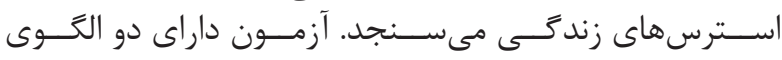

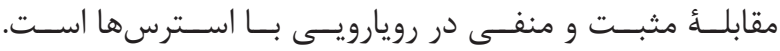

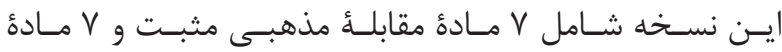

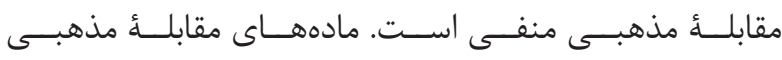

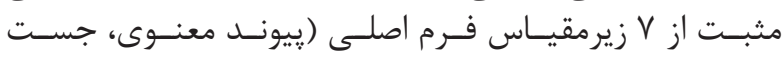

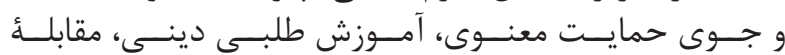

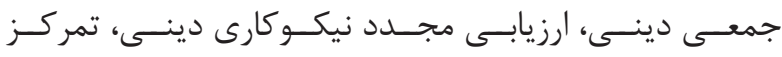

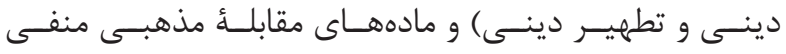

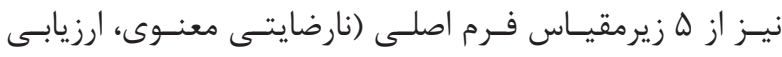

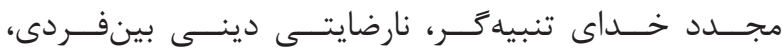

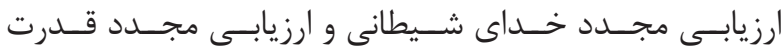

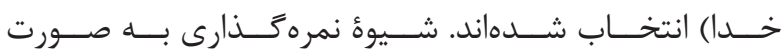

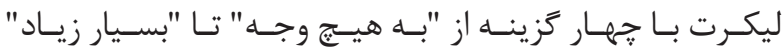

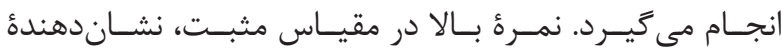

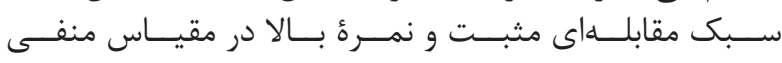

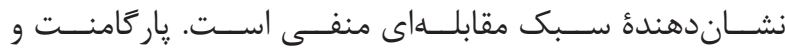

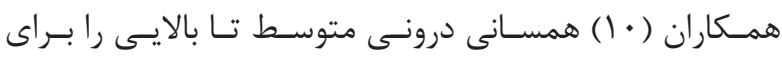

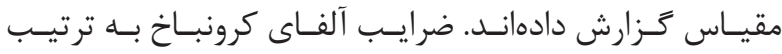

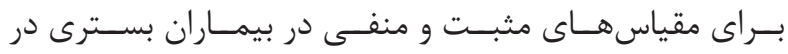

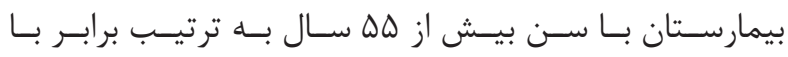

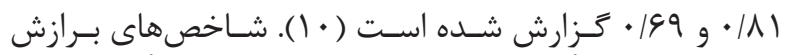

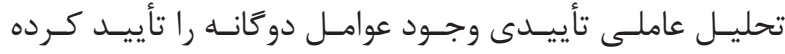

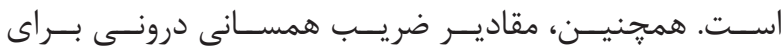

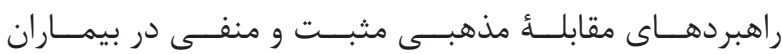

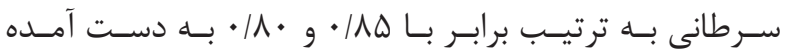

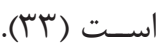

\section{مقياس خودَزارشى هوش معنوى كينَ}

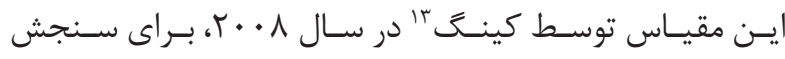

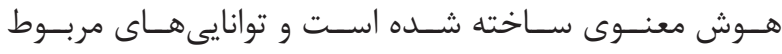

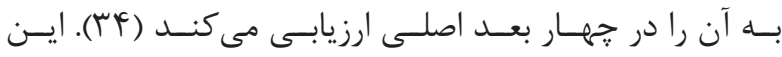

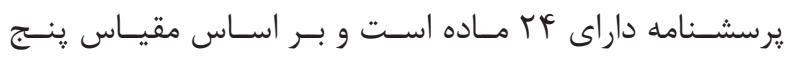

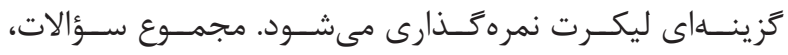

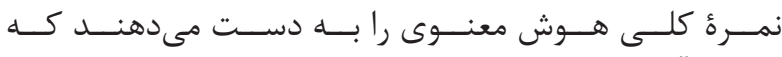

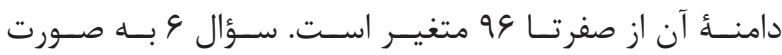




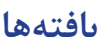

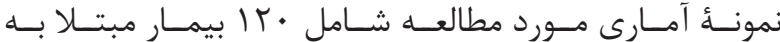

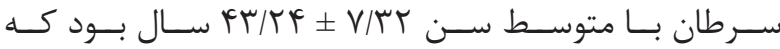

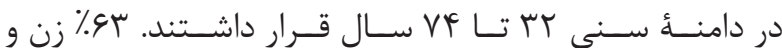
نV

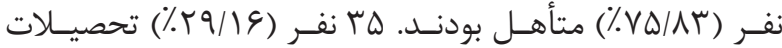

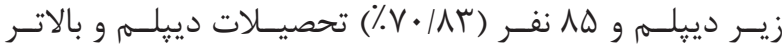

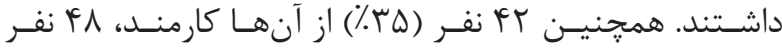

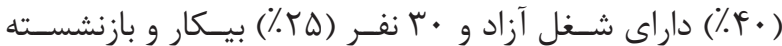

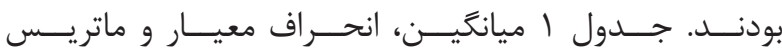

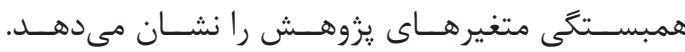

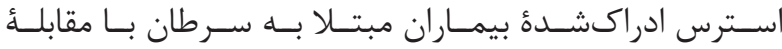

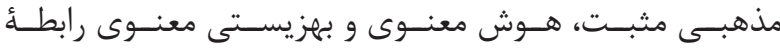

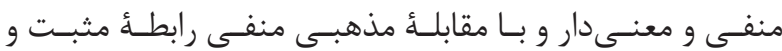

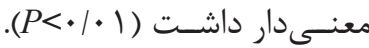

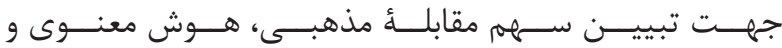

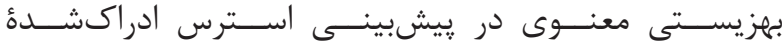

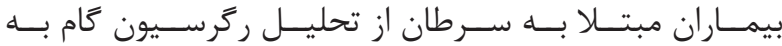

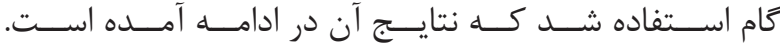

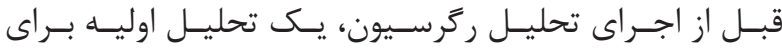

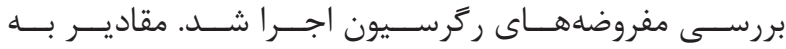

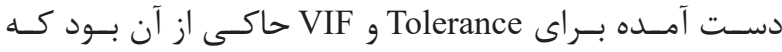

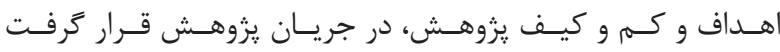

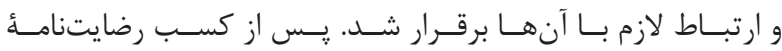

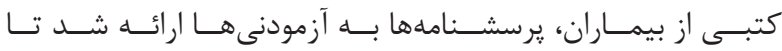

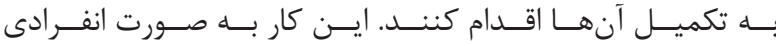

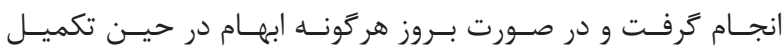

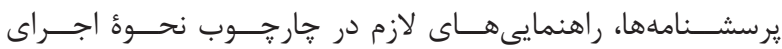

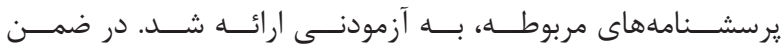

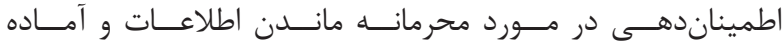

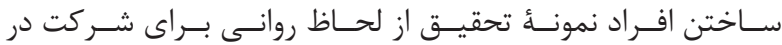

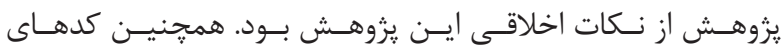

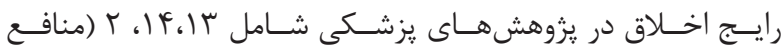

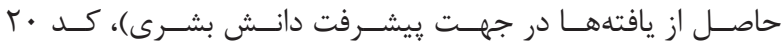

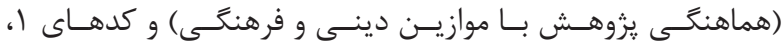

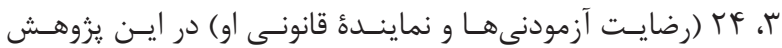

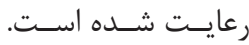
تجزيه و تحليل دادهها

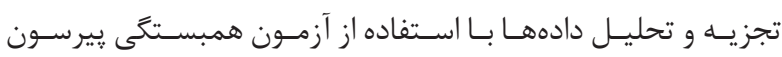

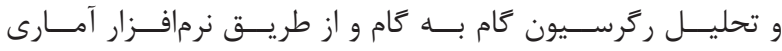

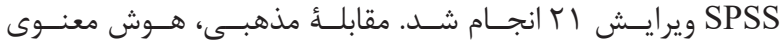

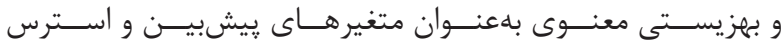

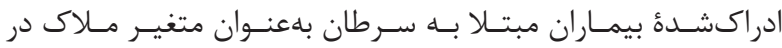

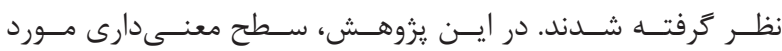
يذيــرش ه • • ن

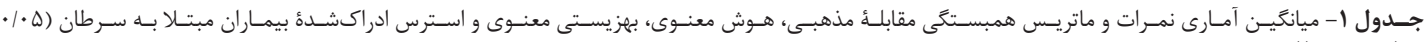

\begin{tabular}{|c|c|c|c|c|c|c|}
\hline$\Delta$ & $p$ & $r$ & $r$ & 1 & ميانتين土|نحراف معيار & متغيرها \\
\hline & & & & 1 & $\mid F / r T \pm \Delta / q r$ & ا. مقابلهُ مذهبى مثبت \\
\hline & & & 1 & $*-\cdot 1 \Delta \Delta$ & $\mid \varphi / \Gamma \wedge \pm \varphi / \|$ & ז. مقابلةُ مذهبى منفى \\
\hline & & 1 & $*-\cdot / 4 \Delta$ & $* * / 4 \varphi$ & $4 \cdot / 99 \pm 1 V / 19$ & "ا. هوش معنوى \\
\hline & 1 & $* \cdot \Delta T$ & $*-\cdot \mid \Delta \varphi$ & $* * / 109$ & $\Delta r / T Y \pm 19 / \cdot F$ & P. بهزيستى معنوى \\
\hline 1 & $* * \cdot \cdot N r$ & $* *-\cdot|\Delta|$ & $* / N$ & $* *-\cdot \mid 94$ & $r q / \cdot r \pm 11 / 11$ & ه. استرس ادراك شده \\
\hline
\end{tabular}

جدول r- مدل نهايیى ركرسيون براى يِيشبينى استرس ادراكشدة بيماران مبتلا به سرطان.

\begin{tabular}{|c|c|c|c|c|c|}
\hline $\boldsymbol{P}$ & $\mathbf{t}$ & $\boldsymbol{\beta}$ & SE & B & متغيرها \\
\hline$\cdot 1 \cdot \cdots$ & II/TFF & - & r/ఎq. & $r \cdot / r V \cdot$ & مقدار ثابت \\
\hline$\cdot 1 \cdots$ & $-0 / 119$ & $-\cdot / \pi \Delta q$ & $\cdot|\cdot|+\mid$ & $-\cdot / 4 \cdot 9$ & بزيستى معنوى \\
\hline$\cdot 1 \cdots$ & $4 / 994$ & • Mth & .1119 & $\cdot / \Delta 9 \Delta$ & مقابله مذهبى منفى \\
\hline$\cdot 1 \cdot \cdot \mathrm{V}$ & - T/VGG & $-\cdot / 1 \mathrm{~V} \cdot$ & $\cdot 1 \cdot \mathrm{r}$ & $-\cdot 11 \cdot 9$ & هوش معنوى \\
\hline $.1 \cdot 11$ & $-r / \Delta \& q$ & $-\cdot / \mathrm{VT}$ & $.11 T G$ & שזr/•- & مقابله مذهبى مثبت \\
\hline
\end{tabular}




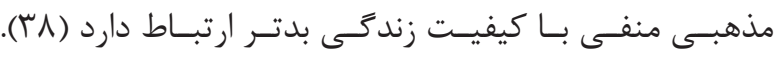

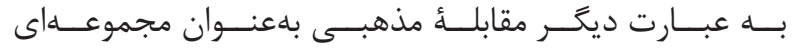

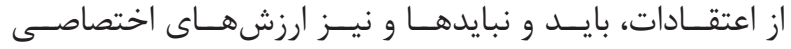

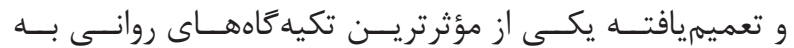

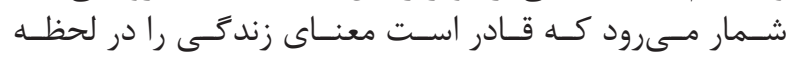

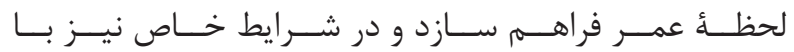

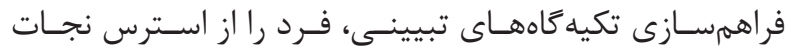

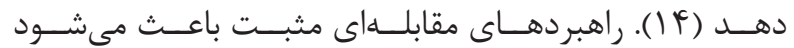

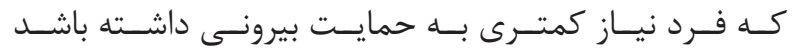

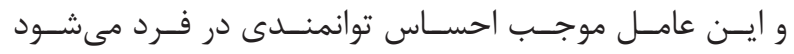

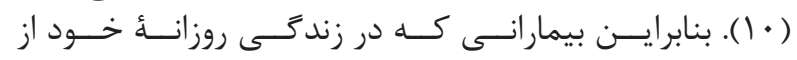

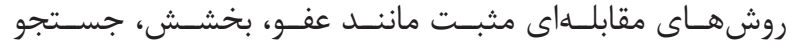

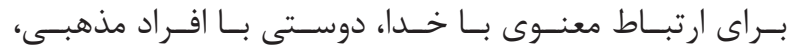

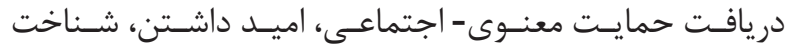

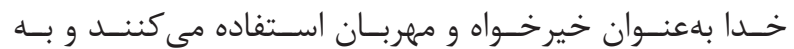

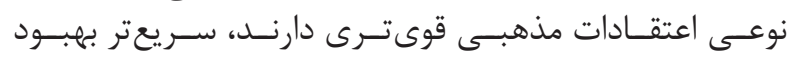

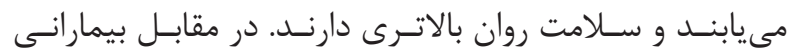

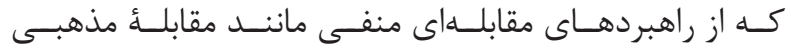

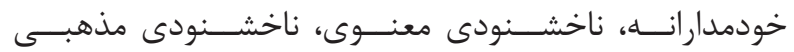

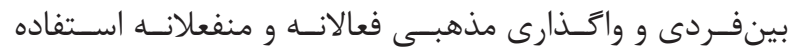

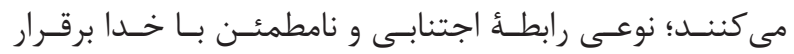

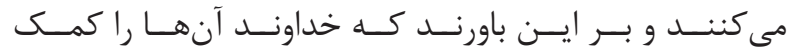

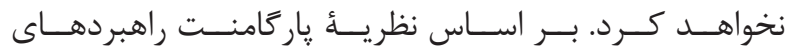

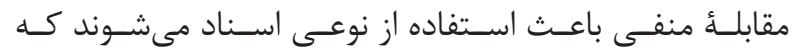

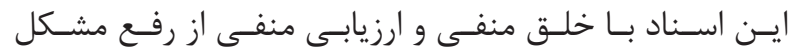

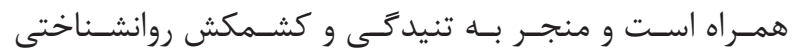

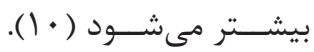

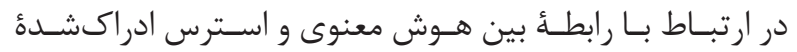

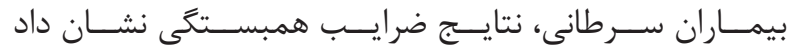

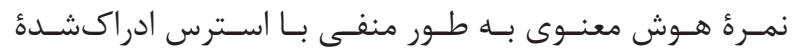

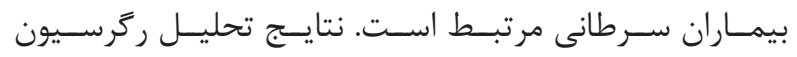

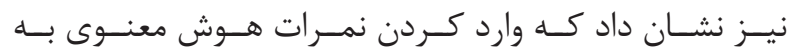

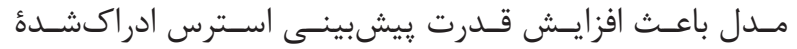

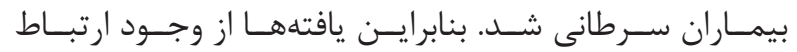

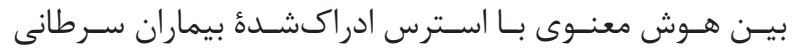

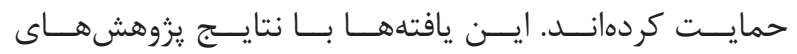

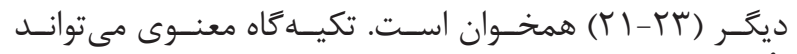

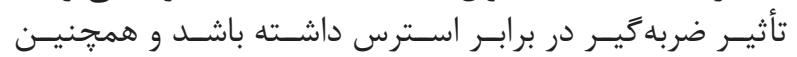

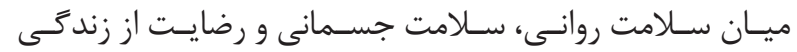

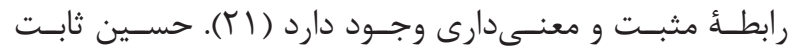

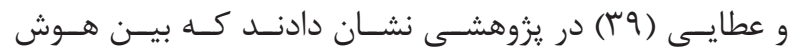

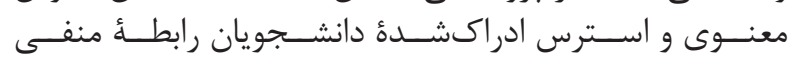

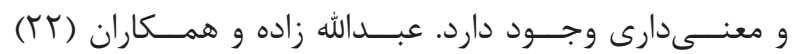

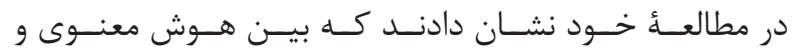

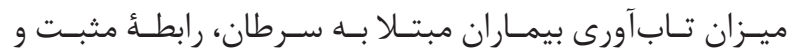

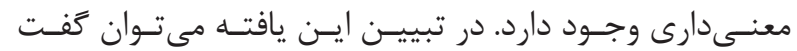

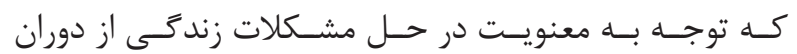

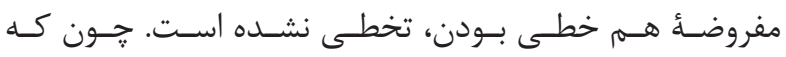

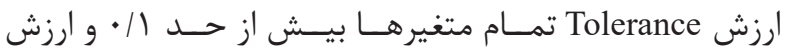

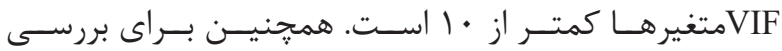

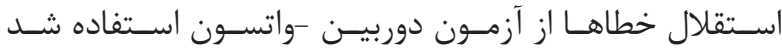

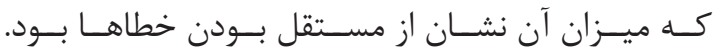

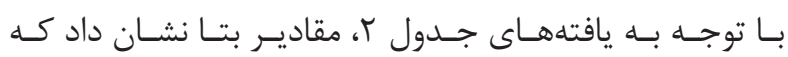

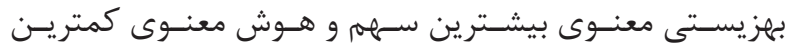

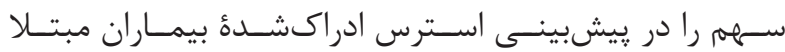

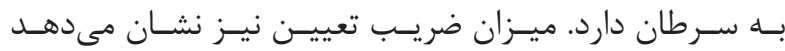

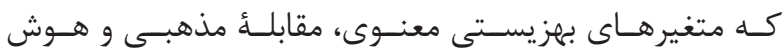

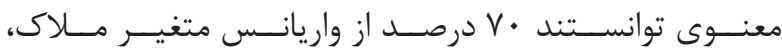

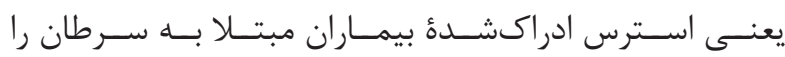

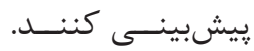

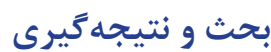

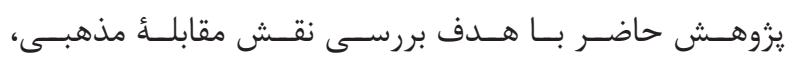

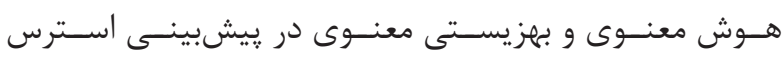

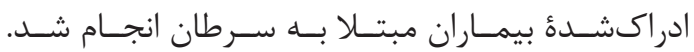

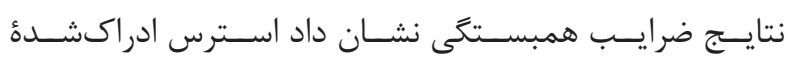

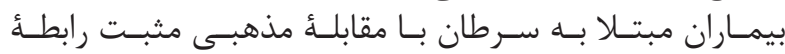

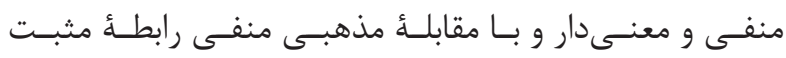

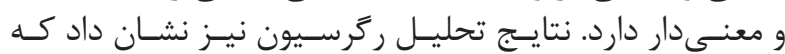

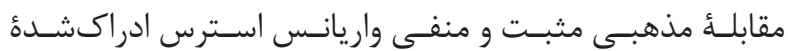

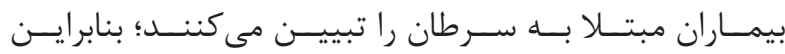

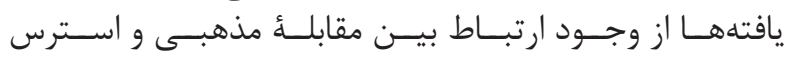

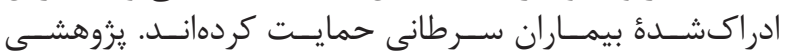

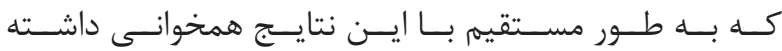

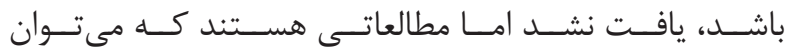

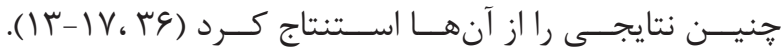

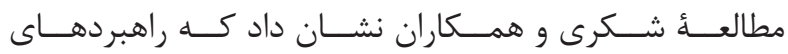

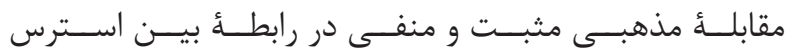

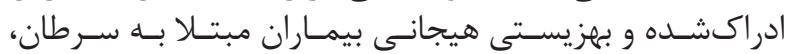

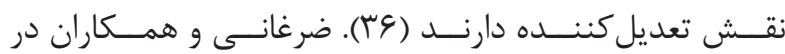

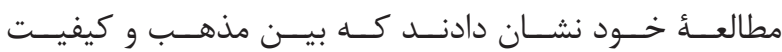

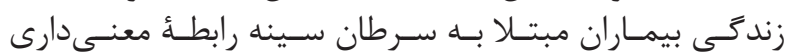

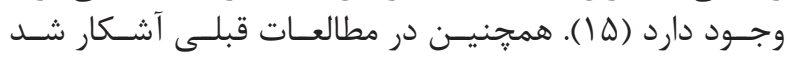

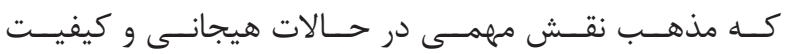

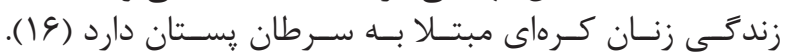

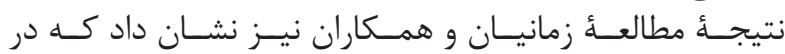

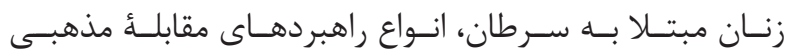

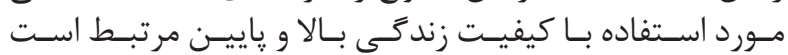

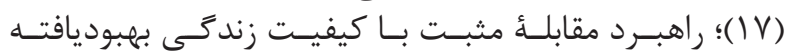

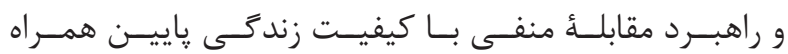

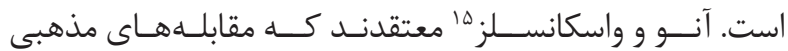

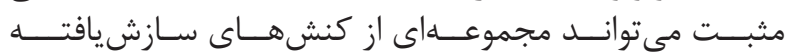

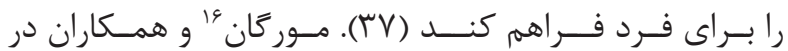

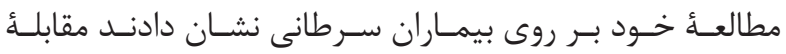

${ }^{15}$ Ano and Vasconcelles

${ }^{16}$ Morgan 


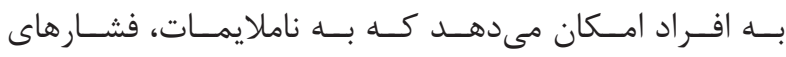

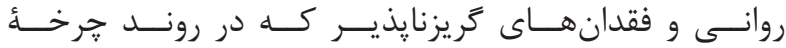

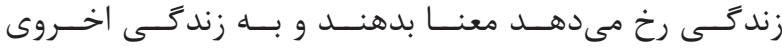

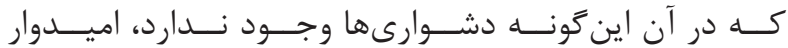

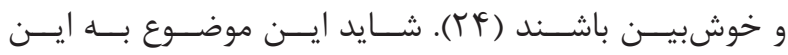

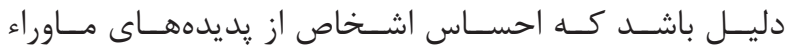

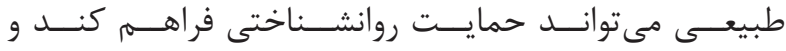

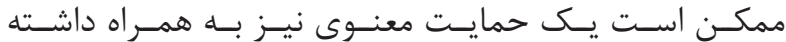

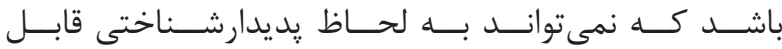

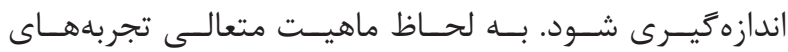

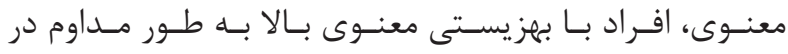

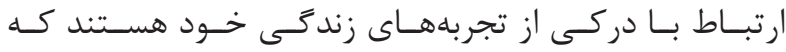

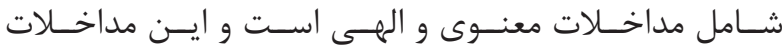

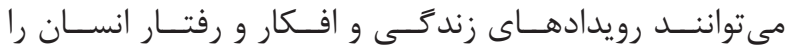

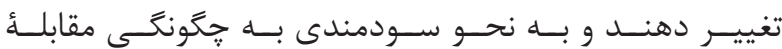

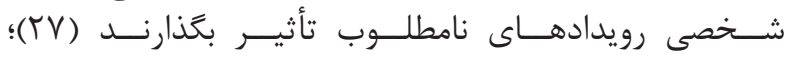

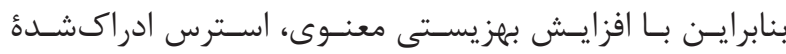

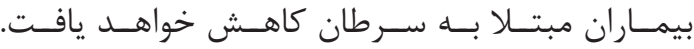

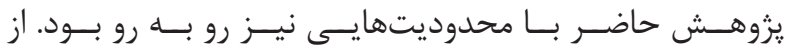

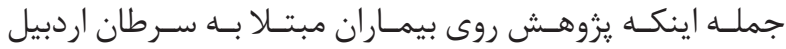

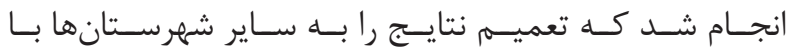

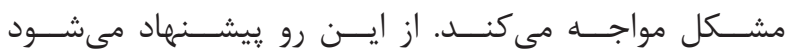

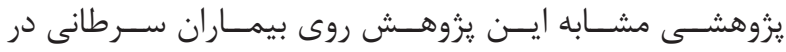

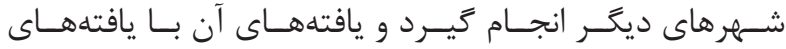

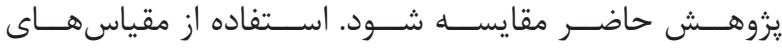

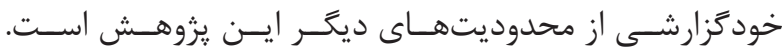

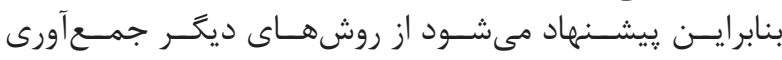

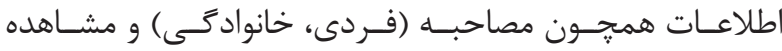

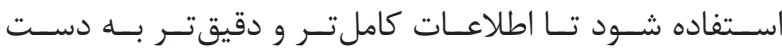

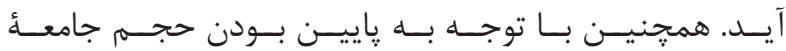

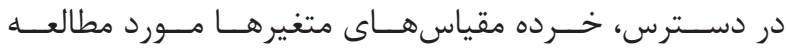

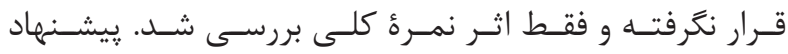

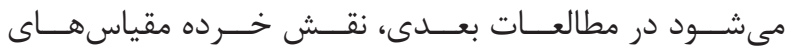

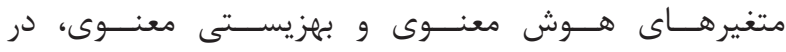

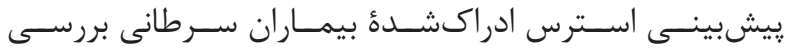

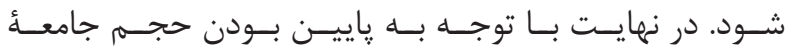

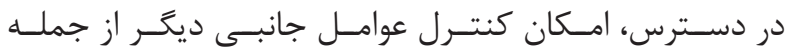

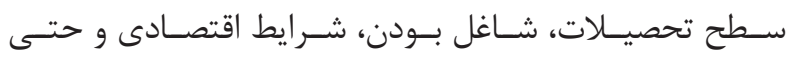

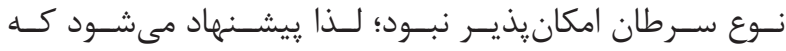

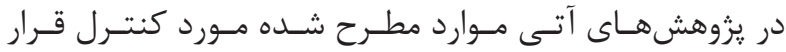

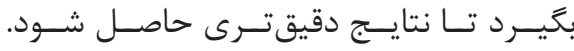

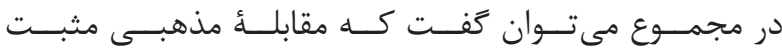

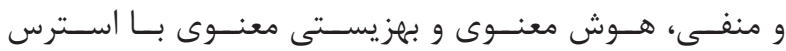

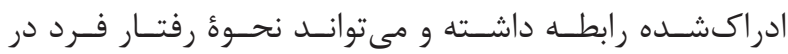

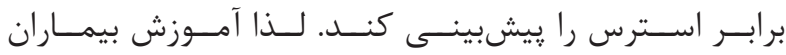

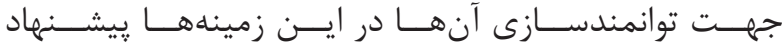

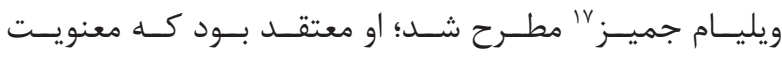

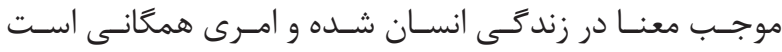

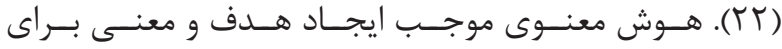

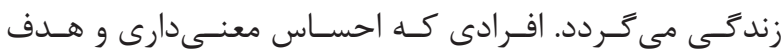

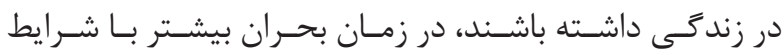

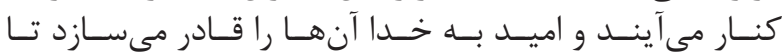

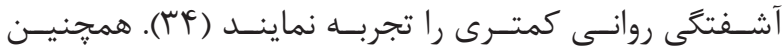

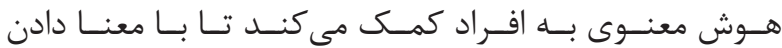

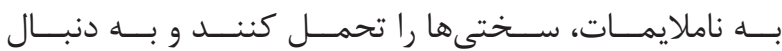

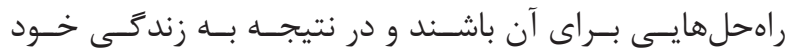

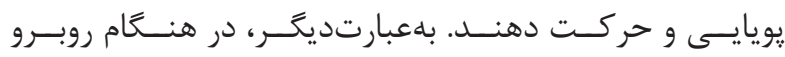

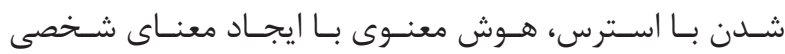

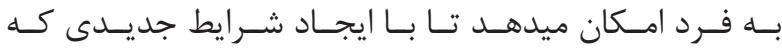

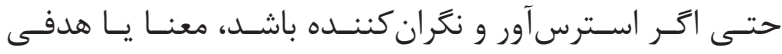

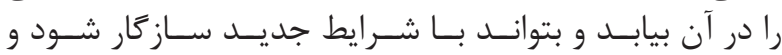

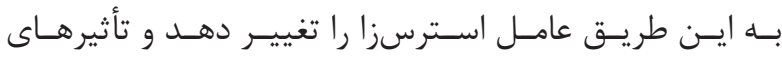

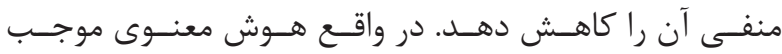

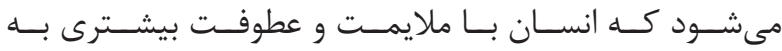

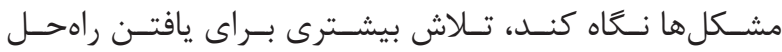

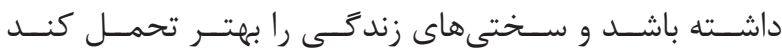

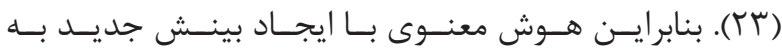

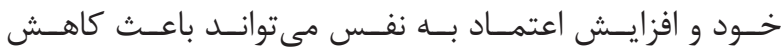

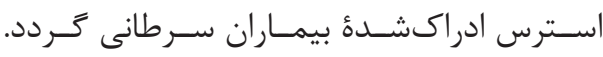

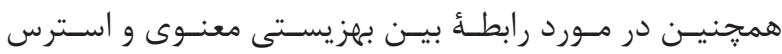

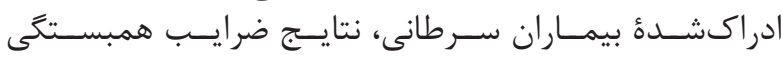

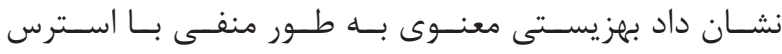

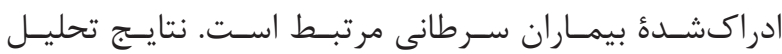

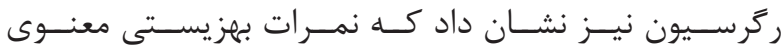

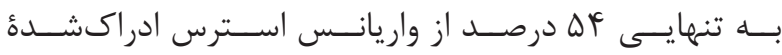

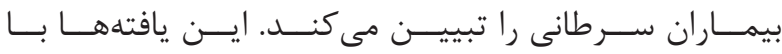

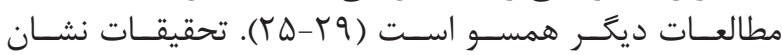

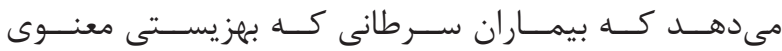

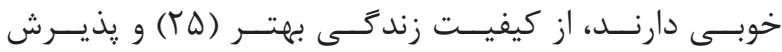

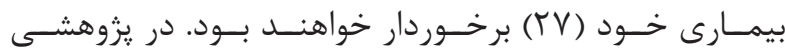

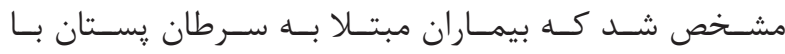

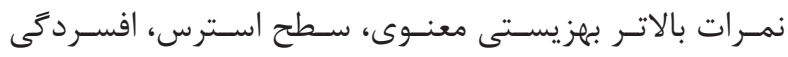

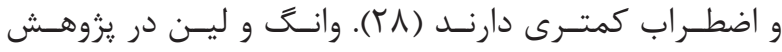

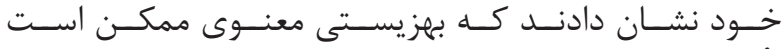

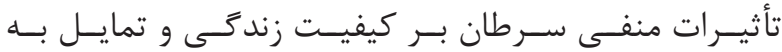

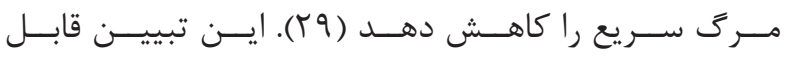

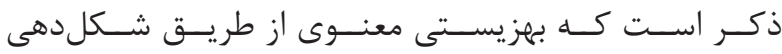

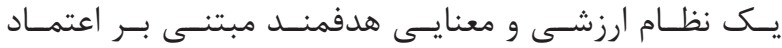

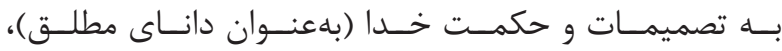

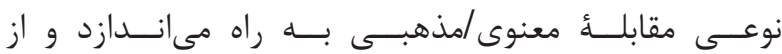

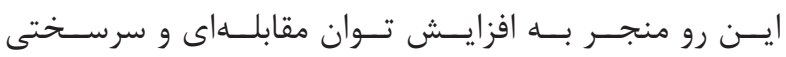

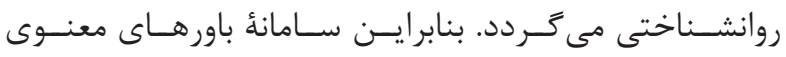




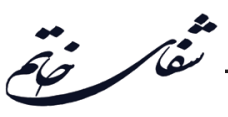

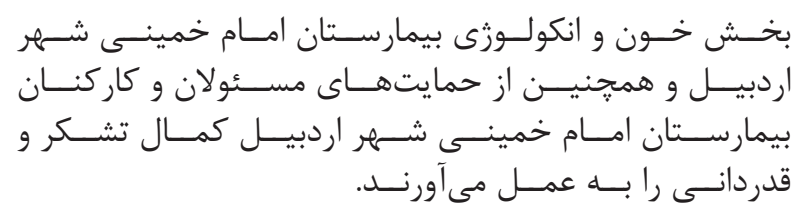

1. Fitzmaurice C, Allen C, Barber RM, Barregard L, Bhutta ZA, Brenner H. Global, regional, and national cancer incidence, mortality, years of life lost, years lived with disability, and disability-adjusted life-years for 32 cancer groups, 1990 to 2015: A systematic analysis for the global burden of disease study. JAMA Oncol. 2017; 3(4):524-48.

2. Pirkhaefi A, Salehi F. Effectiveness of group logo therapy on promotion mental health of women with breast cancer. Health Psychol. 2013; 2(8):61-9.

3. Sieggel RL, Miller KD, Jemal A. Cancer statistics, 2019. CA Cancer J Clin. 2019; 69(1): 7-34.

4. Conley C, Bishop B, Andersen B. Emotions and emotion regulation in breast cancer survivorship. Health Care. 2016; 4(3): E56.

5. Beatty L, Lee C, Wade TD. A prospective examination of perceived stress as a mediator of the relationship between life-events and QOL following breast cancer. Br J Health Psychol. 2009; 14(4):789-804.

6. Penedo FJ, Benedict C, Zhou ES, Rasheed M, Traeger L, Kava BR, et al. Association of stress management skills and perceived stress with physical and emotional well-being among advanced prostate cancer survivors following androgen deprivation treatment. J Clin Psychol Med S. 2013; 20(1): 25-32.

7. Lovell B, Moss M, Wetherell MA. Perceived stress, common health complaints and diurnal patterns of cortisol secretion in young, otherwise healthy individuals. Horm Behav. 2011; 60(3): 301-5.

8. Miceli J, Geller D, Tsung A, Hecht CL, Wang Y, Pathak R, et al. Illness perceptions and perceived stress in patients with advanced gastrointestinal cancer. PsychoOncol. 2019; 28(7):1513-9.

9. Song H, Saito E, Sawada N, Abe SK, Hidaka A, Shimazu T, et al. Perceived stress level and risk of cancer incidence in a Japanese population: the Japan Public Health Center (JPHC)-based prospective study. Sci Rep. 2017; 7(1): 12964.

10. Ano GG, Vasconcelles EB. Religious coping and psychological adjustment to stress: a meta-analysis. J Clin Psychol. 2005; 61(4): 461-80.

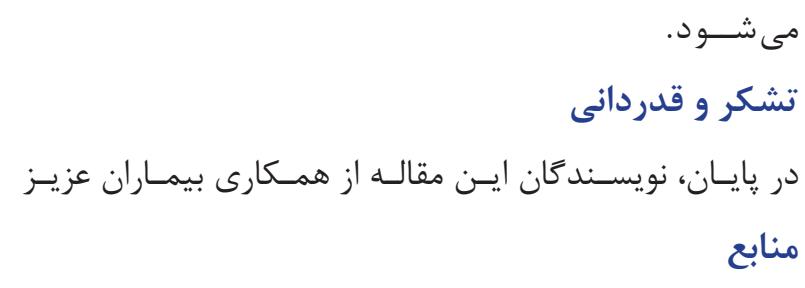

11. Richards AE, Petrie MJ, Chapman KL. Is religious coping a moderator of perceived control and panic symptoms in african american adults? J Black Psychol. 2016; 42(2): 140-59.

12. Pargament KI, Koenig HG, Tarakeshwar N, Hahn J. Religious coping methods as predictors of psychological, physical and spiritual outcomes among medically ill elderly patients: a two-year longitudinal study. J Health Psychol. 2004; 9(6): 713-30.

13. Sajadiyan AS, Haghighat S, Montazeri A, Kazemnejad A, Haghight Sh. Post diagnosis coping strategies patients with breast cancer. Iran J Breast Dis. 2011; 4(1):52-8.

14. Avestan Z, Rahmani A, Heshmati-Nabavi F, Mogadasian S, Faghani S, Azadi A. et al. Perceptions of Iranian cancer patients regarding respecting their dignity in hospital settings. Asian Pac J Cancer Prev. 2015; 16(13): 5453-8.

15. Zargani A, Nasiri M, Hekmat K, Abbaspour Z, Vahabi S. A survey on the relationship between religiosity and quality of life in patients with breast cancer: A study in Iranian muslims. Asia Pac J Oncol Nurs. 2018; 5(2):21722.

16. Jang JE, Kim SW, Kim SY, Kim JM, Park MH, Yoon $\mathrm{JH}$, et al. Religiosity, depression, and quality of life in Korean patients with breast cancer: a 1-year prospective longitudinal study. Psycho- Oncol. 2013; 22(4):922-9.

17. Zamanian H, Eftekhar-Ardebili H, Eftekhar-Ardebili M, Shojaeizadeh D, Nedjat S, Taheri-Kharameh Z, et al. Religious coping and quality of life in women with breast cancer. Asian Pac J Cancer Prev. 2015; 16(17):7721-5.

18. Bediako SM, Neblett EW. Optimism and perceived stress in Sickle-cell disease: The role of an afrocultural social Ethos. J Black Psychol. 2011; 37(2): 234-53.

19. Emmons RA. Spirituality and intelligence: Problems and prospects. Int J Psychol Religion. 2000; 10(1): 5764.

20. Davidson S. Cultivating spiritual intelligence to heal diseases of meaning. Contemp Nurse. 2002; 12(2): 103-5.

21. Calhoun LG, Cann A, Tedeschi RG, McMillan JA. A correlational test of the relationship between 
posttraumatic growth, religion and cognitive processing. J Traumatic Stress. 2000; 13(3): 521-7.

22. Abdollah zadeh R, Moodi M, Allahyari A, Khanjani $\mathrm{N}$. The relationship between spiritual Intelligence and resiliency of patients suffering from cancer in South Khorasan State. Nurs J Vulnerable. 2015; 2(3): 15-24.

23. Moazedyan P, Bagheri M. The spiritual intelligence training to family, affective, social and Physical adjustments in breast cancer patients. Applied Counseling. 2016; 6(1): 89-110.

24. Bai M, Lazenby M. A systematic review of associations between spiritual well-being and quality of life at the scale and factor levels in studies among patients with cancer. J Palliat Med. 2015; 18(3): 286-98.

25. Akalin A, Pinar G. Unmet needs of women diagnosed with gynecologic cancer: an overview of literature. J Palliat Care Med. 2016; 6(2): 1-6.

26. Mahdavi A, Jenaabadi H, Mosavimoghadam SR, Shojaei Langari SS, Gholamali Lavasani M, Madani Y. Relationship between mental, existential, and religious well-being and death anxiety in women with breast cancer. Archives Breast Cancer. 2019; 6(1): 29-34.

27. Zamaniyan S, Bolhari J, Naziri G, Akrami M, Hosseini S. Effectiveness of spiritual group therapy on quality of life and spiritual well-being among patients with breast cancer. Iran J Med Sci. 2016; 41(2): 140-4.

28. Musarezaie M, Esfahani HN, Momeni T, Karimian J. The relationship between spiritual wellbeing and stress, anxiety, and depression in patients with breast cancer. J Isfahan Med School. 2012; 30(195): 922-31.

29. Wang YC, Lin CC. Spiritual well-being may reduce the negative impacts of cancer symptoms on the quality of life and the desire for hastened death in terminally ill cancer patients. Cancer Nurs. 2016; 39(4): 43-50.

30. Delavar A. Theoretical and practical research in the humanities and social sciences. Teharn: Roshd Press; 2011.

31.Cohen S, Kamarck T, Mermelstein R. A global measure of perceived stress. J Health Soc Behav. 1983; 24(4): 385-96.

32. Safaei M, Shokri O. Assessing stress in cancer patients: Factorial validity of the perceived stress scale in Iran. Iran J Psychiatric Nurs. 2014; 2(1):13-22.

33. Abdolkhaleghi M, Shokri O, Safayi M, Salehi R. Psychometric evaluation of the brief religious coping scale for cancer patients. Health Psychol. 2015; 4(1): 113-26.

34. King DB. Rethinking claims of spiritual intelligence: A definition, model and measure [Dissertation]. Canada: Trent University; 2008.

35. Paloutzian, RF, Ellison CW. Loneliness, spiritual well- being, and quality of life. LA Peplau, D Perlman. Loneliness: A Sourcebook foe current theory, research, and therapy. New York: Wiley Interscience; 1982.

36. Shokri O, Salehi R, Safaie M, Abdalkhaleghi M. Perceived stress and emotional well-being: The mediating role of religious coping among Cancer Patients. J Res Psycholo Health. 2014; 8(4): 2-3.

37. Ano GG, Vasconcelles EB. Religious coping and psychological adjustment to stress: a meta-analysis. J Clin Psychol. 2005; 61: 461-80.

38. Morgan PD, Gaston-Johansson F, Mock V. Spiritual well-being, religious coping, and the quality of life of African American breast cancer treatment: a pilot study. ABNF J. 2006; 17(2): 73-7.

39. Hosseinsabet F, Ataei H. The relationship between spiritual intelligence and patience and perceived stress in undergraduate students. J Reas Religion \& Health. 2016; 2(1): 26-33. 